\title{
Controlling the Double Rotary Inverted Pendulum with Multiple Feedback Delays
}

\author{
V. Casanova, J. Salt, R. Piza, A. Cuenca
}

\author{
V. Casanova, J. Salt, R. Piza, A. Cuenca \\ Departamento de Ingeniería de Sistemas y Automática \\ Instituto Universitario de Automática e Informática Industrial \\ Universitat Politécnica de Valencia \\ Camino de Vera s/n 46022 Valencia (Spain) \\ E-mail: \{vcasanov, julian, rpiza, acuenca\}@isa.upv.es
}

\begin{abstract}
:
The aim of this work is the development and implementation of a control structure for the double rotary inverted pendulum, suitable to be used in a Networked Control System environment. Delays are quite common in this kind of systems and, when controlling multivariable plants, it is possible that different delays are applied to the multiple inputs and outputs of them. A control structure that allows compensating individually each one of the multiple loop delays would be useful when one of these delays changes. Inverted pendulums are quite sensitive to delays and for this reason are appropriated plants to be used in these conditions. The control structure is developed modifying the control in no-delay conditions with a generalized predictor able to deal with unstable and non-minimum plants as the chosen one is. The proposed structure has been simulated and implemented to control a real double rotary inverted pendulum.
\end{abstract}

Keywords:Control applications, delay compensation, distributed control, multi-loop control, multi-variable feedback control, transport delay.

\section{Introduction}

The main goal of this paper is to propose a control structure that deals with multivariable plants when different delays are applied to the feedback signals. Consider the scenario in figure 1. A multivariable (MIMO) plant is going to be controlled by a MIMO controller. The plant outputs are feedback into the controller and the control actions are calculated by it, following some predesigned control law. These actions are applied to the plant. If the communication links between controller and plant is ideal, no delays are present. When a real link is used at least one sample time delay must be considered in each communication link. The influence of these delays must be removed to reach the desired behavior: the one in ideal (no-delay) conditions.

In the proposed scenario sensors and actuators (i.e. AD and DA conversions) are supposed to be separated by long distances among them and from the controller. The communication follows different paths for output signals and control actions, suffering different transport delays. The controller used in ideal conditions must be modified to compensate these delays. This is a common situation in networked control systems (NCS) when a shared medium is used to communicate a number of sensors and actuators with the main control. The longer the distance is, the greater the delay must be removed. The delay compensator becomes a MIMO structure to deal with the delay in each pair action/signal.

In wide-area NCS it is usual to have a great number of AD and DA conversions involved in the same control structure. The communication is performed through a shared medium instead of using point-to-point links. This allows to reduce costs (wiring, maintenance...) and 


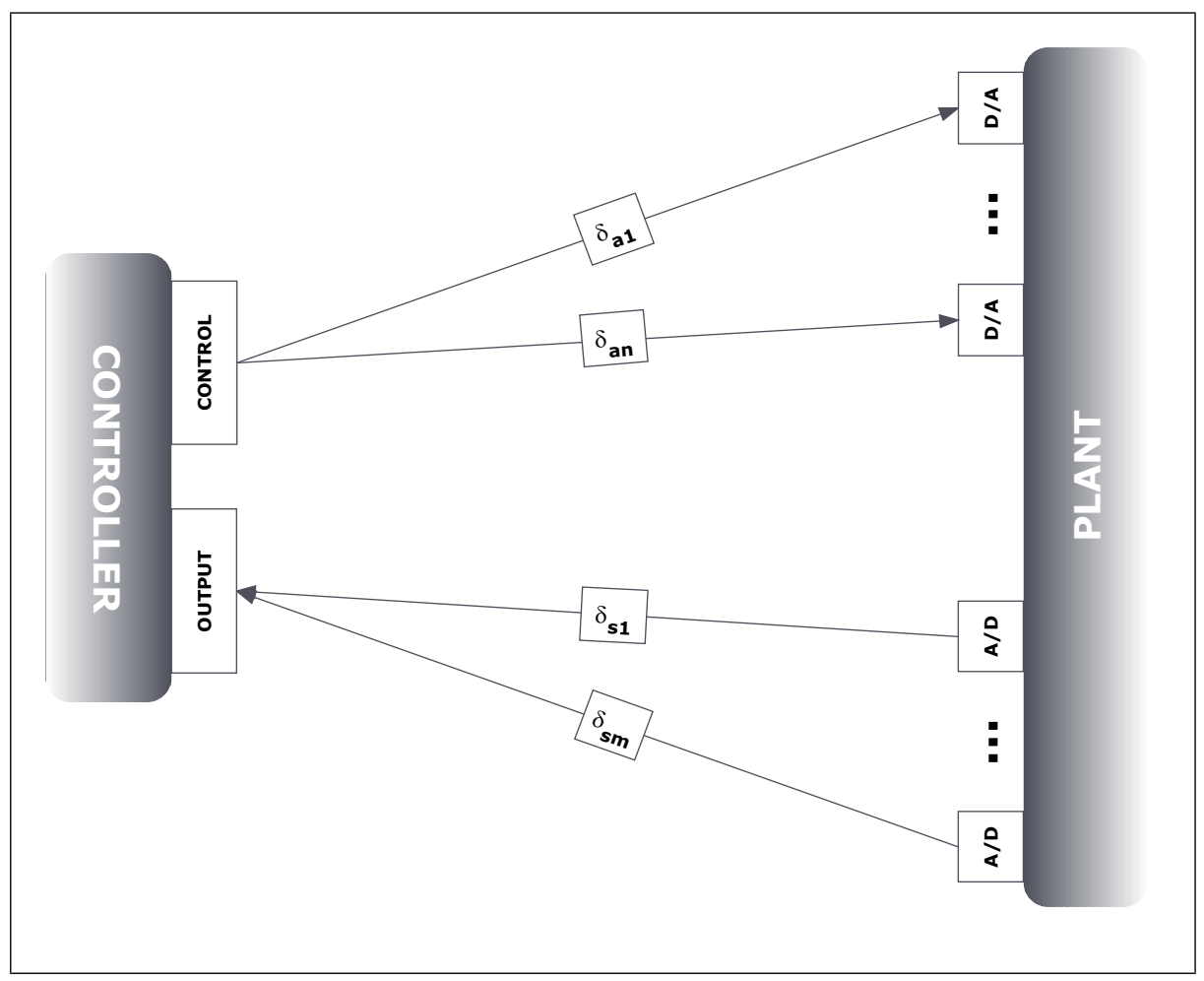

Figure 1: Problem scenario

to increase flexibility of the control system. The main drawbacks are the delay uncertainty and the bandwidth limitation. If there are different distances between sensors/actuators and the controller and/or the priority of the devices attached to the network is different, multiple delays can arise in the control structure.

In the most general case different number of sample time delays are applied to each one of the signals towards the actuators ( $\delta_{a n}$ being $n$ the $n^{\text {th }}$ control action) and to the signals from the sensors $\left(\delta_{s m}\right.$ being $m$ the $m^{\text {th }}$ measured variable). The control structure becomes a collection of $n \times m$ loops each one of them includes a $\delta_{a n}+\delta_{s m}$ sample time delay, from the point of view of the controller (loop delay). This is the delay to be compensated in the controller of each one of these loops in the control structure. By removing the influence of the delays, the ideal behavior is intended to be reached. The goal of this work is to use the same controller, designed without considering the delays, to deal with the problem when they are present.

The presence of multiple delays in a NCS environment has been considered in many descriptions of this kind of systems ( [1], [2], [3], [4], [5], [6], [7]) and several previous works have dealt with this problem. In [8] and [9] there is a description of the problem of multiple delays in NCS when they are smaller than one sampling period. In [10], [11] and [12] a NCS with multiple distributed delays is modeled and an optimal control is proposed. [13] proposes a model of an NCS with multiple successive delay components in the state. [14] deals with the problem of observability and optimal control in these control systems. [15] analyses the maximum allowable delay bound in control loops with network induced delays. [16] considers the problem of multiple delays in NCS from the point of view of fault diagnosis. Some works ( [17], [18]) have considered the problem as a multivariable control design in which multiple input-output delays are included in the plant model. [19] uses an observer to provide state information, comparing the system behaviour with the Smith predictor.

The proposed solution is going to be applied to a classical control problem: the double rotary 
inverted pendulum (also known as double Furuta pendulum, see figure 2). This is a classical textbook example of non-linear, non-minimum phase plant. The plant to be controlled has one input (the control action to the motor) and three outputs (the angular position of the motor shaft, the angular position of the first rod of the inverted pendulum and the angular position of the second rod). The goal of this control problem is to keep the pendulum angles as close to zero as possible, avoiding disturbances and to follow a certain reference with the shaft angle. So, four signals are involved between control and plant and four different time delays are going to be considered. As it is well known, this plant is quite sensitive to delays between controller and plant becoming unstable even with small delays. This sensitivity makes the Furuta pendulum quite appropriated to the proposed environment. The controller used in ideal conditions (i.e. no delays) must be properly modified to remove the delay influence in the feedback loop. In addition, the plant is an unstable and non-minimum phase one which makes more difficult the delay compensation. The single and double inverted pendulums have been widely used as a test-bed for different control strategies ( [20], [21], [22]).

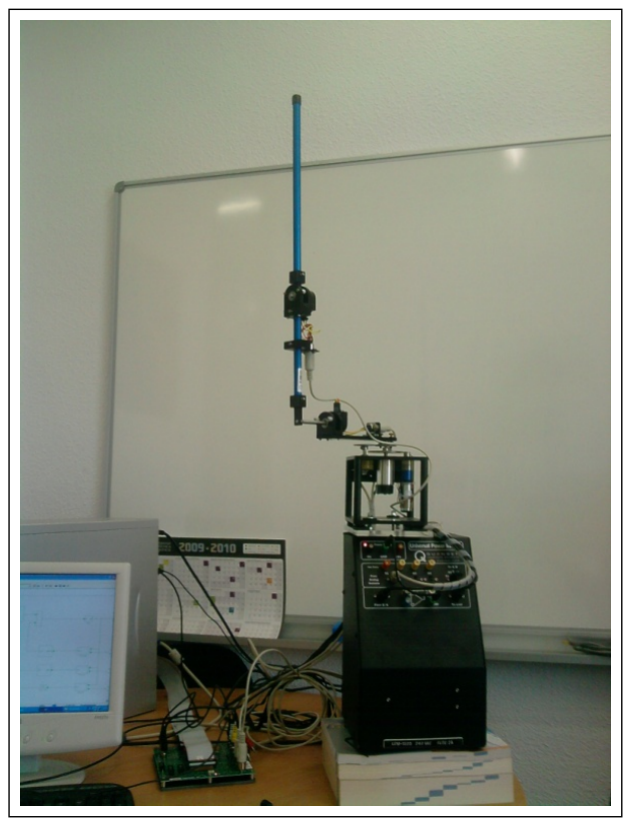

Figure 2: The double rotary inverted pendulum

The paper begins with a description of the plant to be controlled (section 2), followed by the controller used in ideal conditions (section 3). In section 4 the controller is modified to remove the influence of the delays, considering different control-plant delays for each one of the signals involved. Simulated results are presented in these sections to compare the behavior in ideal conditions, the nearly-unstable behavior when delays are included and the behavior when delay influence is removed from the loops. Results from a real plant are presented in section 5 showing how the proposed structure works when real disturbances and noise are present in the system. Finally, in section 6 conclusions and future work are presented.

\section{Double Rotary Inverted Pendulum Model}

The plant that has been chosen to implement the proposed control structure is the double rotary inverted pendulum (double Furuta pendulum) due to its sensitivity to feedback delays. The theoretical model of this plant is described in this section and it is particularized to model 
a real one, the double rotary inverted pendulum developed by Quanser Consulting Inc. (shown in figure 2 in its upwards position).

The equations describing the behavior of the double inverted pendulum can be derived, using the Euler-Lagrange method. A complete development of this model can be found in [23] and [24]. By applying the Euler-Lagrange equation to the Lagrangian (i.e. the difference between kinetic and potential energy) the development of the mathematical model is greatly simplified. Figure 3 shows a schematic representation (top and front view) of the plant. The variables considered in the model are:

- $\theta, \dot{\theta}, \ddot{\theta}$ : Angular position, velocity and acceleration of the motor shaft, around the vertical axis.

- $\alpha, \dot{\alpha}, \ddot{\alpha}$ : Angular position, velocity and acceleration of the first rod, around the motor shaft axis.

- $\gamma, \dot{\gamma}, \ddot{\gamma}$ : Angular position, velocity and acceleration of the second rod, around the motor shaft axis.

- $\tau$ : Torque applied to the motor shaft.

- $u$ : Control action applied to the DC motor (voltage).

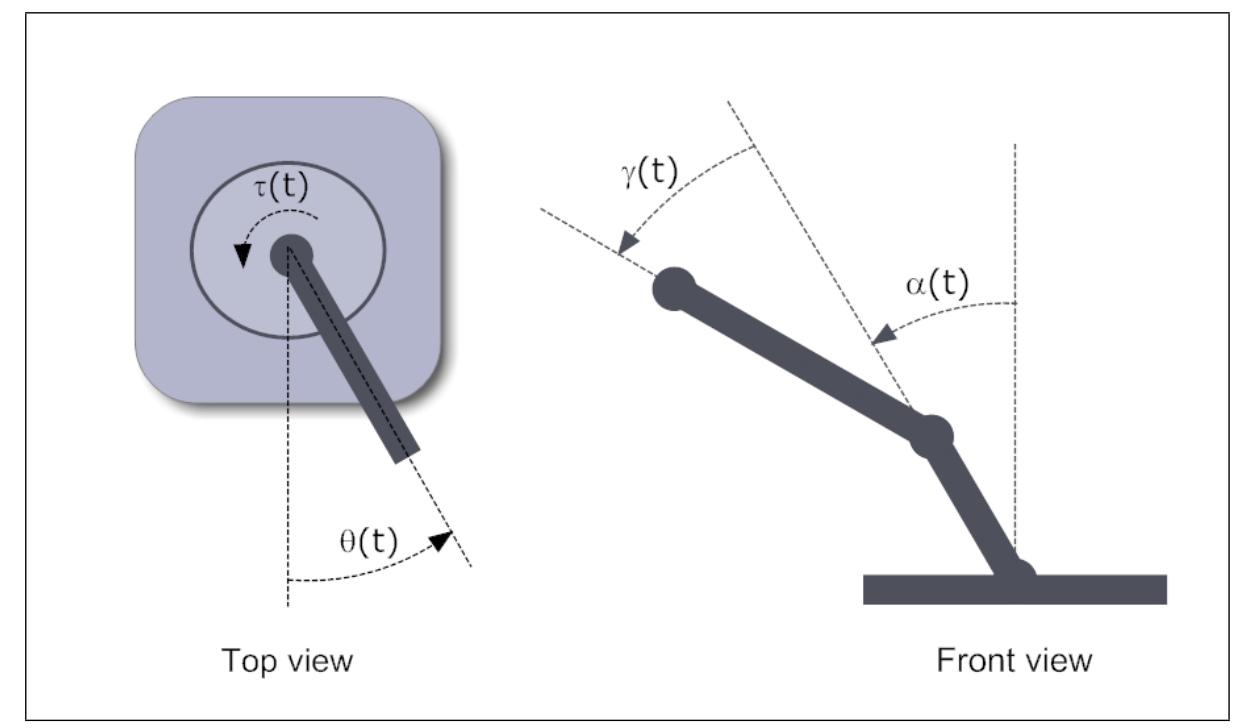

Figure 3: Significant variables.

First, the torque applied by the motor must be related with the angular positions. The three equations defining the non-linear dynamics of the mechanical part of the pendulum can be expressed as follows: 


$$
\begin{array}{r}
\tau(t)=\left[J_{1} L_{1}^{2}\left(m_{2}+m_{3}\right)\right] \ddot{\theta}(t)-\left[L_{1}\left(m_{2} l_{2}+m_{3} L_{2}\right) \cos \alpha(t)\right] \ddot{\alpha}(t)+\left[m_{3} l_{3} L_{1} \cos \gamma(t)\right] \ddot{\gamma}(t)+ \\
+b_{1} \dot{\theta}(t)+\left[L_{1}\left(m_{2} l_{2}+m_{3} L_{2}\right) \sin \alpha(t)\right] \dot{\alpha}(t)^{2}+\left[m_{3} l_{3} L_{1} \sin \gamma(t)\right] \dot{\gamma}(t)^{2} \\
0=-\left[L_{1}\left(m_{2} l_{2}+m_{3} L_{2}\right) \cos \alpha(t)\right] \ddot{\theta}(t)+\left[J_{2}+m_{2} l_{2}^{2}+m_{3} L_{2}^{2}\right] \ddot{\alpha}(t)+ \\
+\left[m_{3} l_{3} L_{2} \cos (\gamma(t)-\alpha(t))\right] \ddot{\gamma}(t)+b_{2} \dot{\alpha}(t)- \\
-\left[m_{3} l_{3} L_{2} \sin (\gamma(t)-\alpha(t))\right] \dot{\gamma}(t)^{2}-\left[g\left(m_{2} l_{2}+m_{3} L_{2}\right) \sin \alpha(t)\right] \\
0=-\left[m_{3} l_{3} L_{1} \cos \gamma(t)\right] \ddot{\theta}(t)+\left[m_{3} l_{3} L_{2} \cos (\gamma(t)-\alpha(t)] \ddot{\alpha}(t)+\left[J_{3}+m_{3} l_{3}^{2}\right] \ddot{\gamma}(t)+\right. \\
+\left[m_{3} l_{3} L_{2} \sin (\gamma(t)-\alpha(t))\right] \dot{\alpha}(t)^{2}+b_{3} \dot{\gamma}(t)-\left[g m_{3} l_{3} \sin \gamma(t)\right]
\end{array}
$$

The parameters (whose values are provided by the manufacturer) in these equations are the lengths, masses, moments of inertia and friction coefficients of the elements in the pendulum.

- $J_{1}$ : Moment of inertia around the center of rotation of the motor arm.

- $J_{2}$ : Moment of inertia around the center of rotation of the first rod of the pendulum.

- $J_{3}$ : Moment of inertia around the center of of the second rod of the pendulum.

- $L_{1}$ : Length of the motor arm.

- $L_{2}$ : Length of the first rod of the pendulum.

- $l_{2}$ : Length between the centers of rotation and gravity of the first rod of the pendulum.

- $l_{3}$ : Length between the centers of rotation and gravity of the second rod of the pendulum.

- $m_{2}$ : Mass of the first rod of the pendulum.

- $m_{3}$ : Mass of the second rod of the pendulum.

- $b_{1}$ : Viscous friction in the joint of the motor arm.

- $b_{2}$ : Viscous friction in the joint of the first rod of the pendulum.

- $b_{3}$ : Viscous friction in the joint of the second rod of the pendulum.

- $g$ : Acceleration of the gravity.

The non-linear model must be linearized to reach a MIMO linear model to be used in the design of the linear controller. The setting point for the linearization will be around $\alpha=0$ and $\gamma=0$, corresponding this values to the upwards position, in which the pendulum is desired to remain stable. The signal derivatives must be zero at the settling point to keep the pendulum stable. In these conditions, the three linear (linearized) equations are:

$$
\begin{array}{r}
\tau(t)=\left[J_{1} L_{1}^{2}\left(m_{2}+m_{3}\right)\right] \ddot{\theta}(t)-\left[L_{1}\left(m_{2} l_{2}+m_{3} L_{2}\right) \ddot{\alpha}(t)+\left[m_{3} l_{3} L_{1}\right] \ddot{\gamma}(t)+b_{1} \dot{\theta}(t)\right. \\
0=-\left[L_{1}\left(m_{2} l_{2}+m_{3} L_{2}\right)\right] \ddot{\theta}(t)+\left[J_{2}+m_{2} l_{2}^{2}+m_{3} L_{2}^{2}\right] \ddot{\alpha}(t)+\left[m_{3} l_{3} L_{2}\right] \ddot{\gamma}(t)+ \\
+b_{2} \dot{\alpha}(t)-\left[g\left(m_{2} l_{2}+m_{3} L_{2}\right) \alpha(t)\right] \\
\left.0=-\left[m_{3} l_{3} L_{1}\right] \ddot{\theta}(t)+\left[m_{3} l_{3} L_{2}\right] \ddot{\alpha}(t)+\left[J_{3}+m_{3} l_{3}^{2}\right] \ddot{\gamma}(t)+b_{3} \dot{\gamma}(t)-\left[g m_{3} l_{3}\right] \gamma(t)\right]
\end{array}
$$

To implement the controller the input must be the voltage applied to the motor that moves the arm that carries the pendulum rods. The relationship between the voltage applied and the torque can be stated from the typical DC motor: 


$$
\tau(t)=\frac{\eta_{g} \eta_{m} K_{g} K_{t}}{R_{m}}\left(u(t)-K_{g} K_{m} \dot{\theta}(t)\right)
$$

The mechanical parameters in this fourth equation are:

- $\eta_{g}$ : Gearbox efficiency.

- $\eta_{m}$ : Motor efficiency.

- $K_{g}$ : Total gear ratio.

- $K_{t}$ : Motor torque constant.

- $K_{m}$ : Motor back-EMF constant.

- $R_{m}$ : Motor armature resistance.

Solving the system of four equations, the linear continuous state-space model of the plant to be controlled can be obtained. The model must have the motor voltage as input signal and the three angular positions as output signals. The three angular velocities are not included as outputs because they are not going to be measured in the real plant. The development is omitted here for space reasons. As the goal is to control a real pendulum, the model has to be particularized with the real parameters (dimensions, masses, inertias, frictions, motor constants...). These parameters have been provided by the manufacturer. This information can be reached from the Quanser documentation [25].

As the control is going to be designed and implemented in discrete time, the continuous model must be discretized. The sample time is going to be $10 \mathrm{~ms}$, small enough to control the plant and large enough to become unstable with a couple of sample time delays.

This model is the one to be used when designing, first, the controller in ideal conditions and, after that, the predictor to remove the influence of the loop delays. The behavior, with and without the delays, must be similar in both, simulated and real systems. The delay compensation is going to be different (because the delays are different) for the three controlled variables. The MIMO system can be divided into three SISO systems one for each of the output signals, $(\theta, \alpha$ and $\gamma$ as described before):

$$
\begin{array}{r}
G_{\theta}(z)=\frac{\theta(s)}{U(s)}=\frac{0.0015763(z+0.9446)(z-0.9432)(z-0.8953)(z-1.061)(z-1.145)}{(z-1)(z-0.9716)(z-0.9077)(z-1.084)(z-1.18)(z-0.7665)} \\
G_{\alpha}(z)=\frac{\alpha(s)}{U(s)}=\frac{0.0018286(z+0.9477)(z-0.912)(z-1)(z-1.114)}{(z-0.7665)(z-0.9077)(z-0.9716)(z-1.084)(z-1.18)} \\
G_{\gamma}(z)=\frac{\gamma(s)}{U(s)}=\frac{-0.0019976(z+0.9538)(z-0.9968)(z-1)^{2}}{(z-0.7665)(z-0.9077)(z-0.9716)(z-1.084)(z-1.18)}
\end{array}
$$

As can be seen these transfer functions describe the dynamical behaviour of unstable and non-minimum phase systems. The MIMO model will be used in the following section to design the controller in ideal conditions. The three SISO models will be used in section 4 to design the predictors for delay compensation purposes. 


\section{Control Structure in No Delay Conditions}

To reach the desired behavior, a Linear-Quadratic Regulator (LQR) controller is going to be used control the plant in absebtia of delays. The controller must keep the angles of the two rods as close to zero as possible (unstable equilibrium upwards position), canceling the unavoidable disturbances. In addition the system must follow a certain reference for the motor shaft angle. The control structure is not robust enough to cope with delays, becoming unstable, even though they are very small. This ideal control will be appropriately modified to remove loop delays in the following section. In order to improve the steady-state error of the shaft position and the robustness of the controller, the six-state model is increased including an integration term. With this term the motor shaft can follow step references with zero error, even with the small dead zone present in the real plant. The integral of $\theta(t)$ will be computed in the controller from the measured angle. This augmented state is now:

$$
\tilde{X}=\left(\begin{array}{lllllll}
\theta(t) & \alpha(t) & \gamma(t) & \dot{\theta}(t) & \dot{\alpha}(t) & \dot{\gamma}(t) & \int \theta(t) d t
\end{array}\right)^{T}
$$

The six-state MIMO model obtained in the previous section is modified, including this new state:

$$
\dot{X}=A X+B u \longrightarrow \dot{\tilde{X}}=\left(\begin{array}{cc}
A & 0 \\
0 & 0
\end{array}\right) \tilde{X}+\left(\begin{array}{c}
B \\
0
\end{array}\right) u
$$

Using Matlab lqr command, the seven feedback control gains for the seven states from the augmented plant model are:

$$
K=\left(\begin{array}{lllllll}
1.2824 & -42.4077 & -101.7583 & 1.8735 & -11.8712 & -11.5716 & 0.3162
\end{array}\right)
$$

Although is not included in the model and, so, not considered when designing the controller, the real control action saturated \pm 10 volts. This saturation has been included in the simulation model. However, in ideal conditions and when the delay is compensated, the control action always remains in this range and the saturation can be ignored.

This controller needs the seven states as inputs to generate the control action to be applied to the plant. In the real plant only the first three of them will be available, by means of sensors measuring the angular positions. There will not be sensors to measure the other states (angular velocities and integral error). These states are going to be computed in the control structure using a filtered discrete derivative and a discrete integrator. So, the controller becomes a three inputs-one output discrete system to be implemented in the control device.

As it has been described in the introduction, the three inputs of the controller are arriving through different paths and different delays are applied to them. To compensate the delays independently it will be useful to decompose the controller into three sub-controllers, each one of them concerned for one of the three measured angles. The transfer functions of these individual controllers are as follows:

$$
\begin{array}{r}
C_{\theta}(z)=\frac{U_{\theta}(z)}{\theta(z)}=K(1)+K(4) \operatorname{Drv}(z)+K(7) \operatorname{Int}(z)=K(1)+K(4) \frac{50 z-50}{z-0.6065}+K(7) \frac{0.01}{z-1} \\
C_{\alpha}(z)=\frac{U_{\alpha}(z)}{\alpha(z)}=K(2)+K(5) \operatorname{Drv}(z)=K(2)+K(5) \frac{50 z-50}{z-0.6065} \\
C_{\gamma}(z)=\frac{U_{\gamma}(z)}{\gamma(z)}=K(3)+K(6) \operatorname{Drv}(z)=K(3)+K(6) \frac{50 z-50}{z-0.6065}
\end{array}
$$


Using $\operatorname{Drv}(z)$ and $\operatorname{Int}(z)$ as discrete derivator and integrator respectively, all the states can be considered measurable. The control structure with these three sub-controllers is shown in figure 4 .

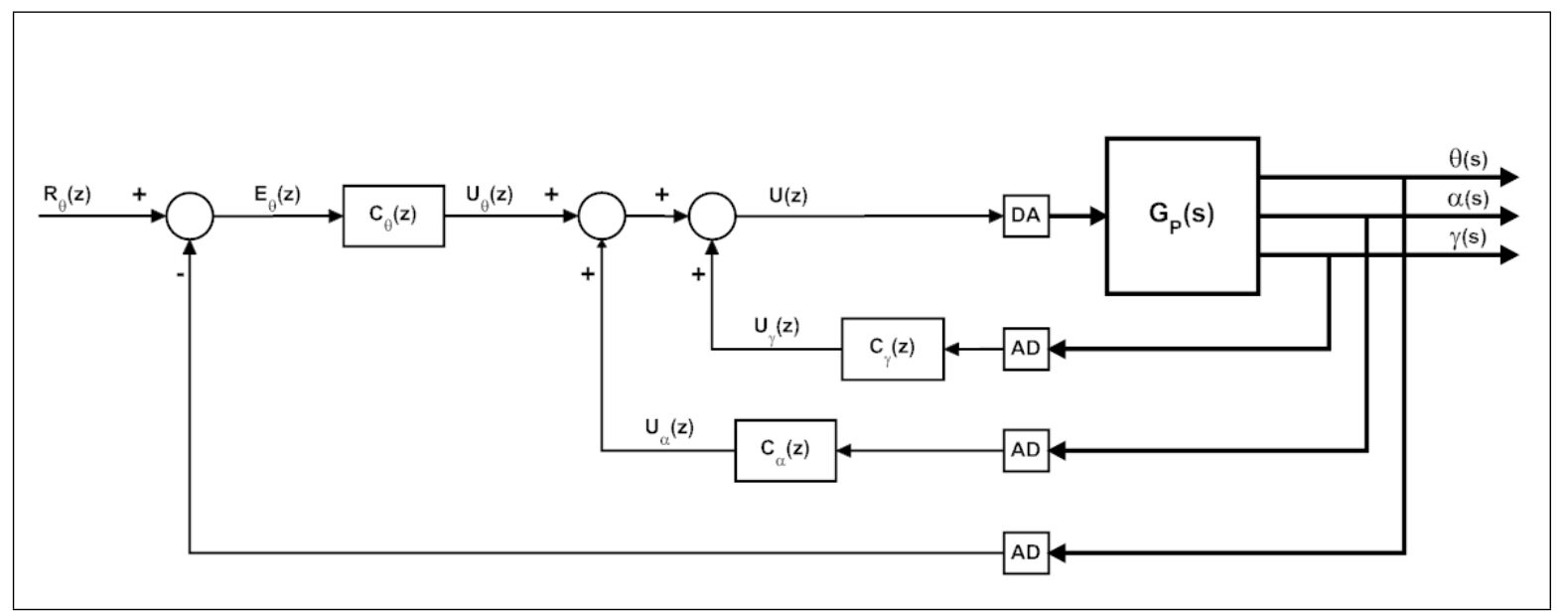

Figure 4: Control structure without delay compensation.

The inner loop tries to keep $\gamma$ angle as close to zero as possible, avoiding disturbances. The middle loop does the same with $\alpha$ angle. The combination of these two loops keep the pendulum in the upwards position as desired. The outer loop tries to follow the desired reference with $\theta$ angle and drives the integral of the error to zero. As the three plant-to-control signals and the control-to-plant signal are separated, different delays will be applied to the four communication links to study its influence in the system performance. With the described control structure, three sub-control actions are generated and the addition of them is the control action to be sent and then applied to the plant.

Closing the loop with the model from the previous section and the gain matrix from the LQR, the simulated results are shown in figures 5 and 6 . A \pm 45 degrees step reference has been applied to be tracked by the shaft angle $(\theta)$ and a small random noise has been added to the measured pendulum angles $(\alpha$ and $\gamma)$. No loop delay is present. Figure 5 depicts the output variables ( $\theta, \alpha$ and $\gamma$ angles in degrees). As can be seen, the reference is followed properly (with zero steady-state error) by the shaft angle and the pendulum angles are kept close to zero, despite the noise and the changes in the main reference. Figure 6 shows the control actions of the three sub-controllers and the addition, which is the final control action to be applied to the plant. Note that $\alpha$ and $\gamma$ control actions are clearly influenced by the noise, added to the measured angles.

\section{Control Structure with Delay Compensation}

Delays in the communication between controller and plant are a well-known source of instability. This is critical when controlling processes as unstable as the Furuta pendulum is. The influence of the delay in the control loop must be removed to assure that the system remains stable. Figure 7 shows the behavior of the three controlled angles when a single sample time delay is included in the control action (with \pm 10 volts saturation in the control action as in the real plant). Another sample time delay in one of the feedback signals makes the system completely unstable. The sensitivity of the plant to loop delays makes this plant the ideal one for the work in this paper. The proposal is to modify the original delay-free controller to handle with delays even when they are different in the signals transmitted to and from the plant. 


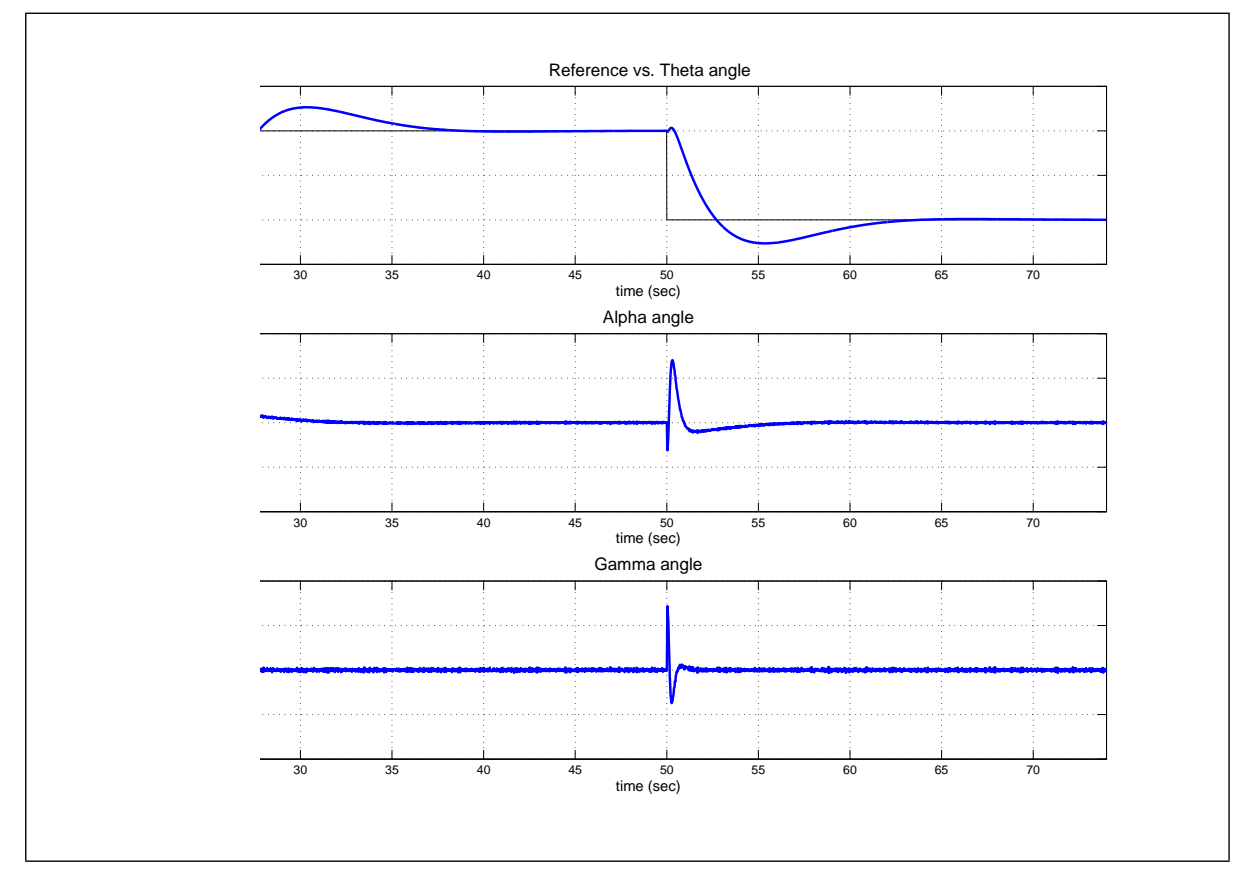

Figure 5: Theta, alpha and gamma angles: ideal conditions.

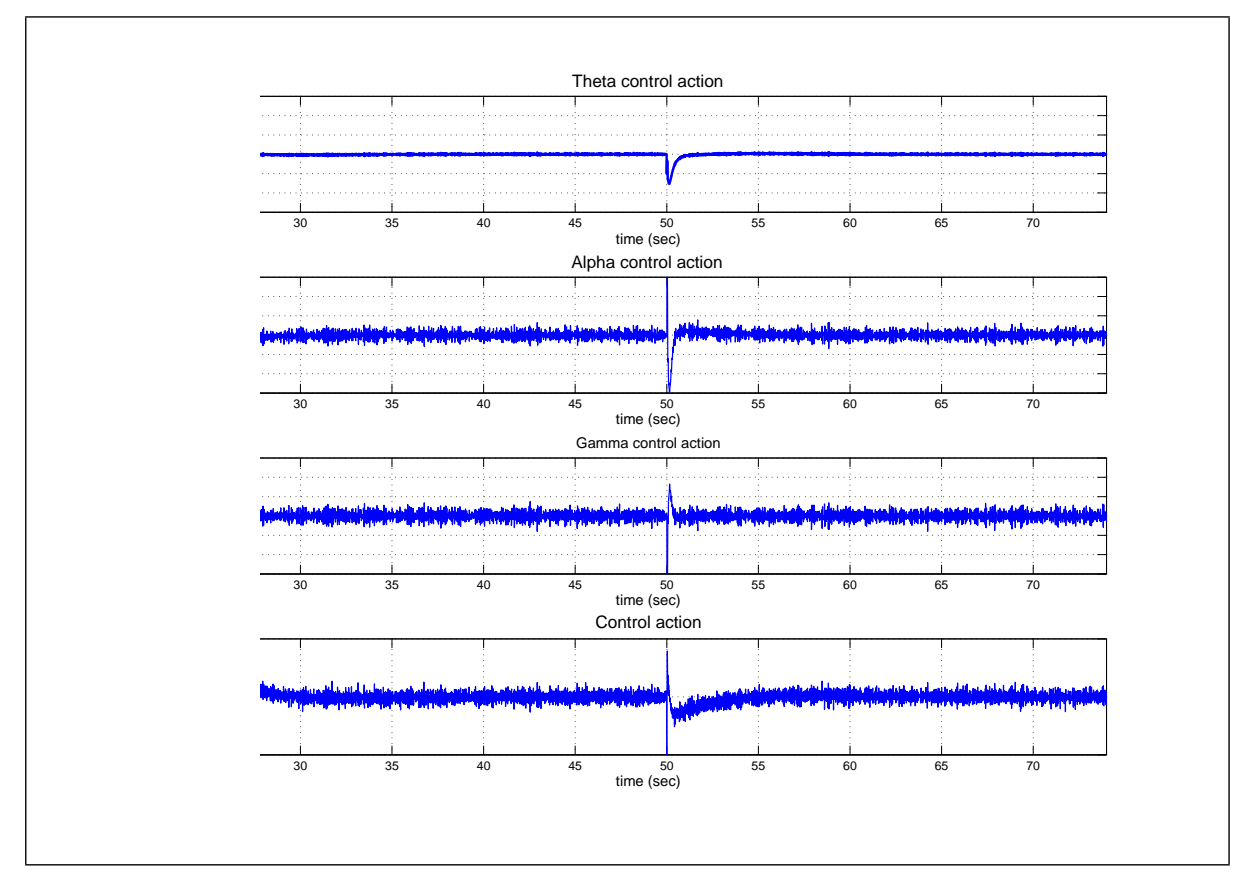

Figure 6: Control actions: ideal conditions. 


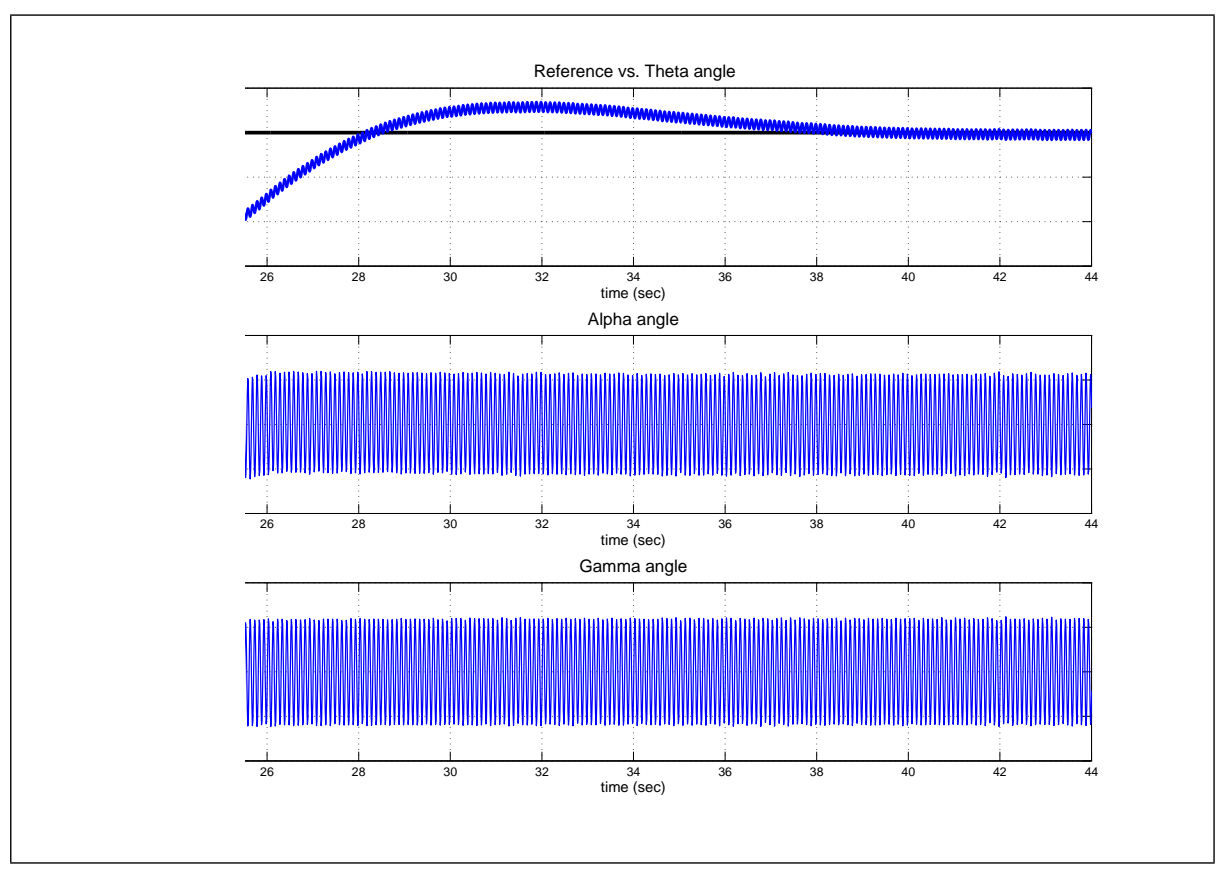

Figure 7: Theta, alpha and gamma angles: delayed.

Smith predictor has been traditionally used to overcome delays for stable plants without integral behavior. Several modifications ( [26], [27], [28]) of the original idea have been proposed to be used for multivariable plants, with different input-output delays. The limitation is that they usually cannot be used with non-minimum phase, unstable plants with integral behavior. The classical Smith predictor has been modified ( [29], [30], [31]) to deal with unstable plants. If all the controlled variables (i.e. all the loops in the control system) have the same delay, one of these modified predictors could be used.

The plant to be controlled in this paper is an unstable and non-minimum MIMO (one input and three outputs) plant but it can be easily decomposed into three SISO plants. In addition, in the scenario considered in this work, each one of the SISO plants is influenced by a different loop delay to be compensated. Parameter $\delta_{u}$ is the number of sample time delays for the control action (controller-to-plant signal). Parameters $\delta_{\theta}, \delta_{\alpha}$ and $\delta_{\gamma}$ are the delays for the shaft and pendulum angles (plant-to-controller signals). The most general case is given when the four delays are different, as shown in figure 4, there are three loops in the systems. The loop delay (from the output to the input of the controller) for each one of the loops is given by the addition of the control action delay and the corresponding output signal delay. These are the delays to be compensated in each one of the sub-controller. Only constant and integer multiple of the sample time delays are considered in this work but the idea can be extended to fractional and variable delays.

In [32] and [33] a delay compensation is proposed, suitable to be used with unstable and nonminimum phase SISO plants. The predictor proposed and described in these references (Generalized predictor, GP, figure 8) is stable for unstable/stable, minimum/non-minimum plants.

The inputs of the GP are the plant input (control action) and output (controlled variable). The output of the GP is the predicted signal. Depending on the quality of the plant model used to design the predictor, the predicted signal will be equal to the controlled variable without the delay. It is the same behavior than with the classical Smith predictor but, in this case, it can be used with the transfer functions of the inverted pendulum. From the (unstable nonminimum phase) transfer function and the known loop delay the GP can be designed. This GP 


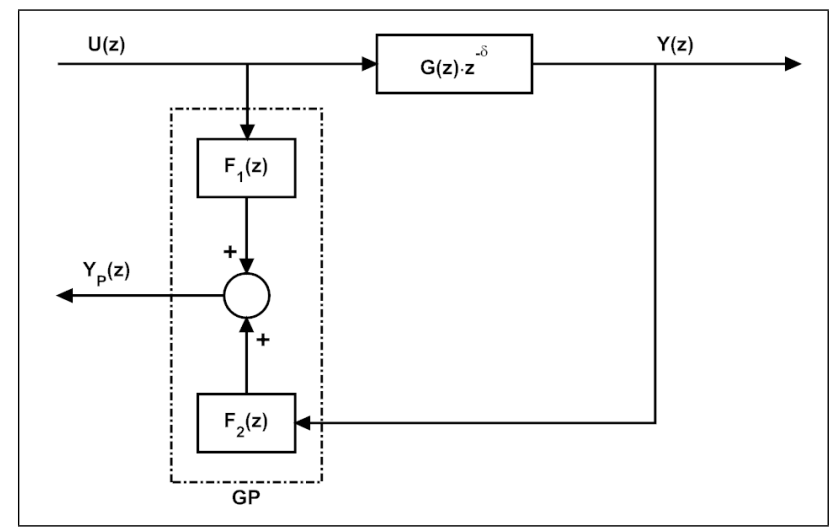

Figure 8: Generalized predictor.

will provide the information to be used by the controller, removing the influence of the delay in the feedback. The predicted (undelayed) output will be used instead of the measured one, by the same controller designed in ideal (no-delay) conditions. As usual, the system behavior depends on the uncertainty of the model and the presence of significant disturbances.

To generate this predicted signal, transfer functions $F_{1}(z)$ and $F_{2}(z)$ must be calculated. Being $G(z)$ the plant to be controlled, this transfer function can be divided in two parts:

$$
G(z)=G_{M P}(z) G_{N M P}(z)
$$

$G_{M P}(z)$ is a transfer function including al the poles (stable and unstable) and the minimum phase zeros. $G_{N M P}(z)$ is a polynomial including the non-minimum phase zeros. $G_{M P}(z)$ can be expressed as a polynomial quotient or as a minimum order state space model:

$$
G_{M P}(z)=\frac{N_{M P}(z)}{D_{M P}(z)}=C_{M P}\left(z I-A_{M P}\right)^{-1} B_{M P}
$$

A new transfer function can be defined, including the delay $(\delta)$ in the minimum phase one:

$$
G_{M P}^{*}(z)=\frac{N_{M P}^{*}(z)}{D_{M P}(z)}=C_{M P}\left(z I-A_{M P}\right)^{-1} A_{M P}^{\delta} B_{M P}
$$

From these definitions the two transfer functions of the GP can be calculated as follows:

$$
\begin{array}{r}
F_{1}(z)=\left(C_{M P} \sum_{i=1}^{\delta} A_{M P}^{\delta} B_{M P} z^{-i}\right) \\
G_{N M P}(z) \\
F_{2}(z)=\frac{N_{M P}^{*}(z)}{N_{M P}(z)}
\end{array}
$$

The predicted output (i.e. the estimation of the plant output without delay) is calculated by the GP as follows:

$$
Y_{P}(z)=U(z) F_{1}(z)+Y(z) F_{2}(z)
$$

In the proposed scenario, three nested loops are present in the system. Three different loop delays are considered and had to be compensated. Three different predictors must be designed and included in the control structure. Figure 9 shows the control structure including the three 


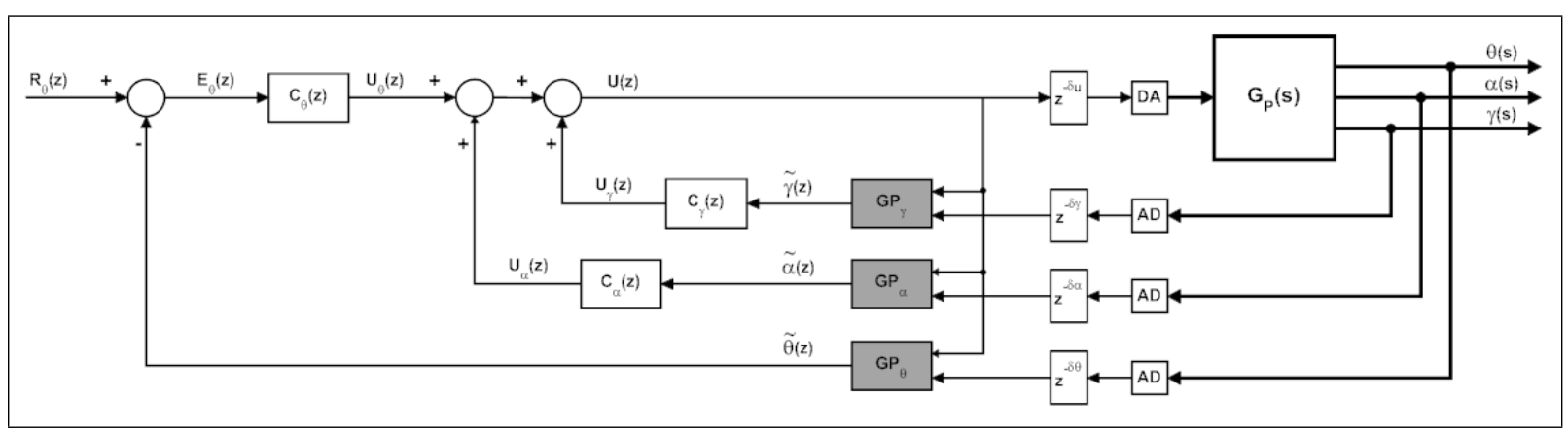

Figure 9: Control structure with delay compensation.

predictors to provide undelayed information for the controllers. Note that the controllers in this structure are the same used in figure 3, when no delay was present in the system.

To compare the information provided by the plant (measured) and by the GP's (predicted), figure 10 shows a detail of the measured angles (in radians) compared with the predicted ones. Measured (red) and predicted (blue) signals can be easily distinguished as the measured is delayed signal respect to the predicted one. Undelayed signals are not equal to the plant outputs due to the noised included in the simulation. It can be seen that the delay compensated by the predictors are different for $\theta, \alpha$ and $\gamma$ angles. In the simulation one sample period delay has been included in the control action, two sample delays in the $\gamma$ angle, three sample delays in the $\alpha$ angle and four sample delays in the $\theta$ angle. So, the loop delay for the $G P_{\gamma}$ predictor is $\delta_{u}+\delta_{\alpha}=3$ and for $G P_{\alpha} \delta_{u}+\delta_{\theta}=4$. These delays can be seen in the time between predicted and measured signals.

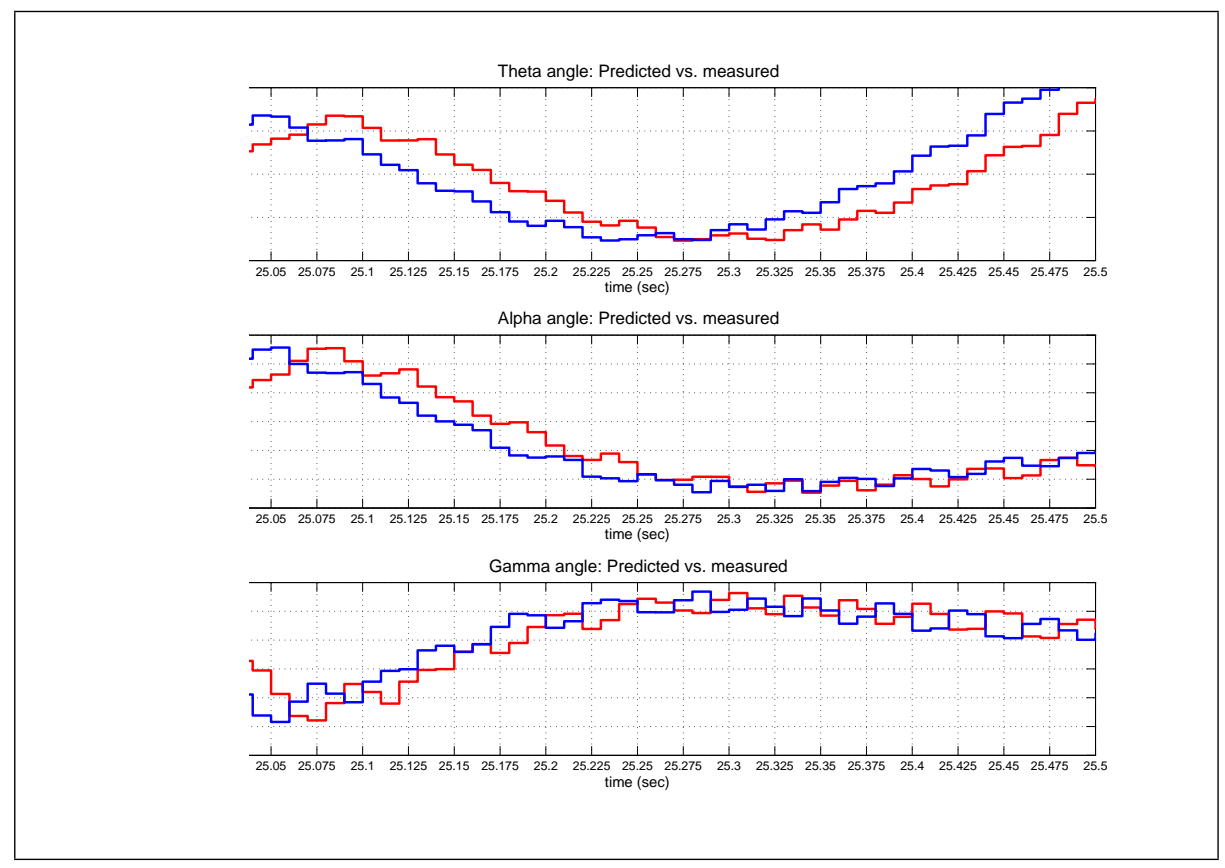

Figure 10: Measured and predicted angles.

The delay-free predicted signals are used as inputs for the three independent controllers to generate the control action to be applied to the plant. Figures 11 and 12, show simulation results in these conditions. Comparing these results with the ones in figures 5 and 6 , it can be seen that the system recovers the ideal conditions behavior, even with different loop delays in the signals 
between controller and plant. Real results are presented in the following section.

\section{Experimental Results}

The results presented in the previous section have been obtained from a simulation model. It is easier to see how the control structure works in simulated results without (unbounded) noise and/or uncertainty. Nevertheless, the work would remain uncompleted if not implemented over a real plant to reach real results.

The proposed structure has been implemented to control a real plant, the Quanser double rotary inverted pendulum (figure 2). The plant is endowed with a potentiometer to measure the shaft angle $(\theta)$ and a couple of incremental encoders to measure the angles of the two rods of the pendulum $(\alpha$ and $\gamma)$. It has also a tachometer to measure the shaft angular velocity but it has not been used in the implementation.

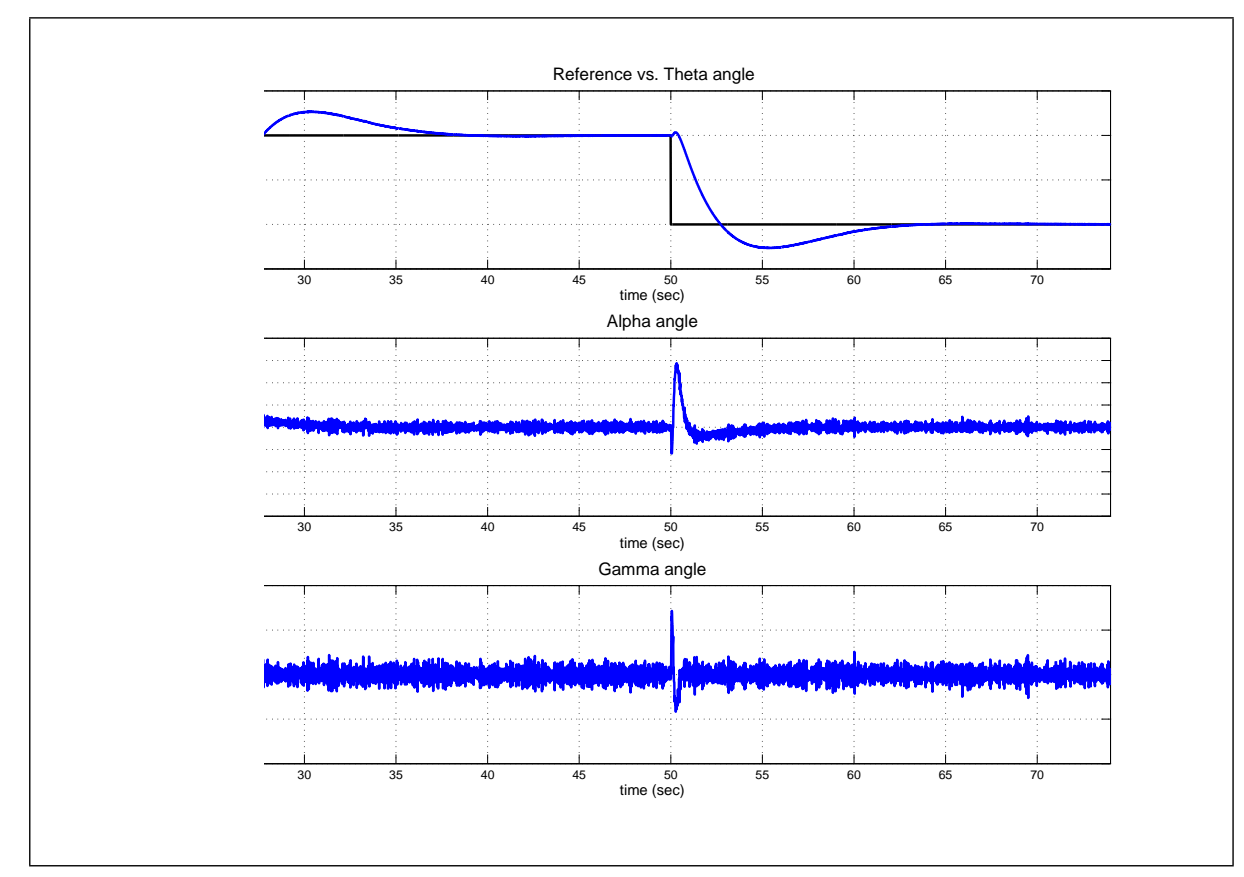

Figure 11: Measured angles: delayed + compensated.

The signal applied to the plant saturates at \pm 10 volts but the action calculated by the controller is almost always inside this range. The aim is to consider a plant in which the different (and long enough) distance between each one the three sensors and the device implementing the control introduces non-negligible delays. However, with the dimensions of the used prototype, the multiple delays between controller and plant had to be simulated and included in the control structure.

Matlab/Simulink Real-Time Windows Target has been used to implement the control structure, using Quanser Q4 board for the A/D and D/A conversions. As noted in section 2, a sample time $T=10 \mathrm{~ms}$ has been used, being large enough for real-time requirements. With this sample time, two sample time delays in the inner loop of the proposed structure are enough to make the system unstable. Figure 13 shows the real measured angles when the delays appear, being the pendulum in its upwards stable position. The conventional control cannot bear this delay and the pendulum falls.

To overcome this delay influence, three GP's has been included in the control structure, as 


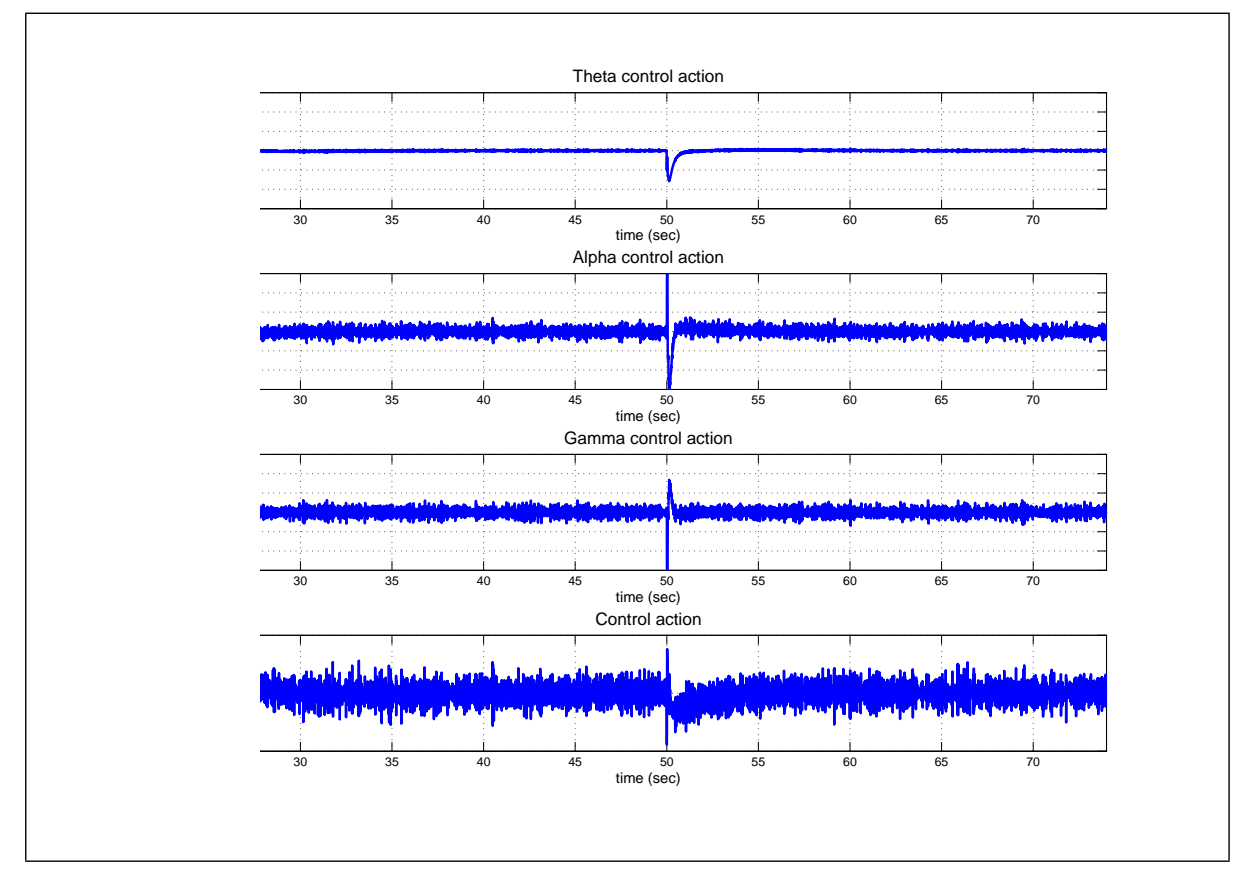

Figure 12: Control actions: delayed + compensated.

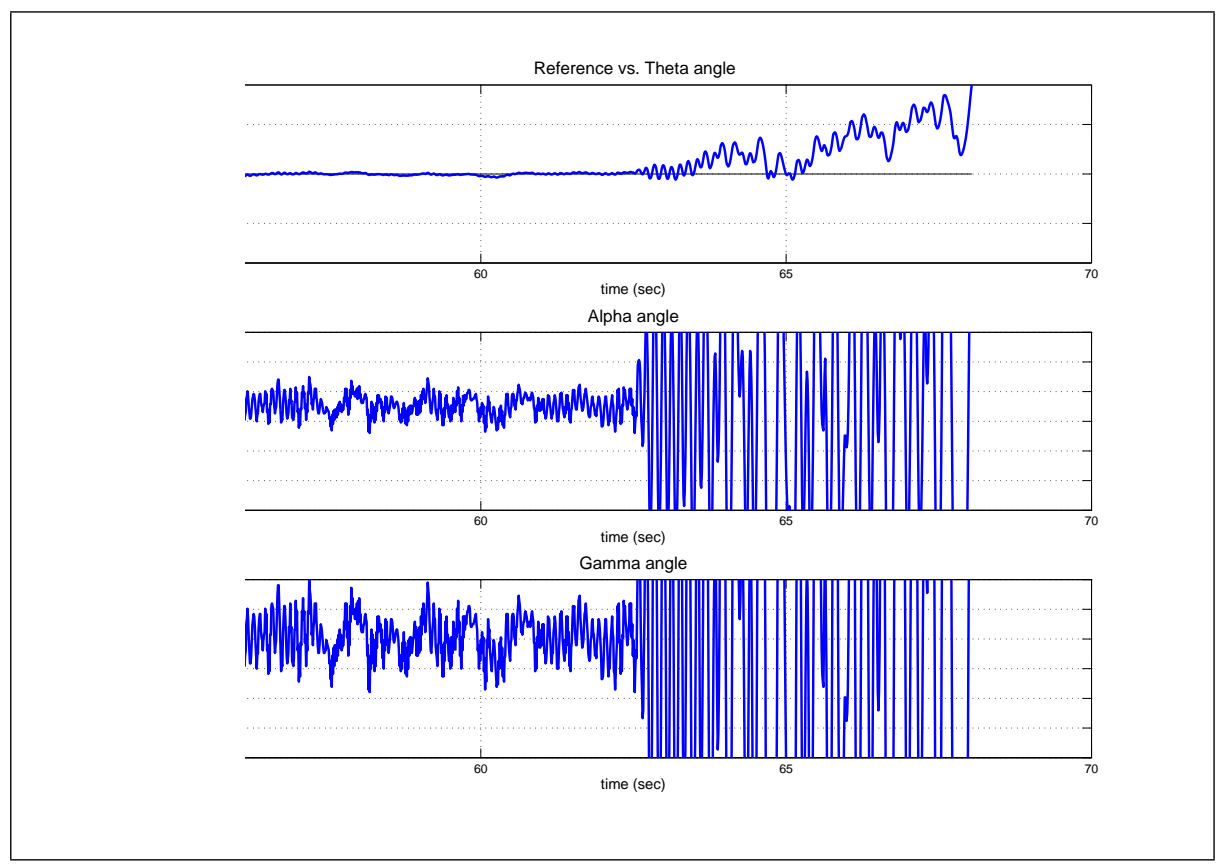

Figure 13: Real results: unstable behavior. 
shown in figure 9.As in the simulation results, the GP's have been calculated to compensate delays $\delta_{u}=1, \delta_{\theta}=3, \delta_{\alpha}=2$ and $\delta_{\gamma}=1$. The predictor in the inner loop, $G P_{\gamma}$ offers a prediction for the $\gamma$ angle with a $z^{-2}$ delay. In the middle loop, $G P_{\alpha}$ has been designed for a $z^{-3}$ delay. Finally, in the outer loop $G P_{\theta}$ predicts the shaft angle removing a $z^{-4}$ delay. With these delays in the loops, the control is absolutely unstable if the measured angles are used. Figure 14 shows the results in these conditions, using the predicted signals instead of the measured ones.

As can be seen, the shaft angle is following a \pm 45 degrees reference and keeps the rods angles close to zero, to hold the pendulum in its upwards position, despite noises and disturbances. It is quite difficult to see differences between these results and the ones in ideal conditions (without delays) because the behavior depends deeply on the initial setup to put the plant in its working point. Figure 15 shows the behavior with some mechanical disturbances in the second rod angle. The control structure keeps the pendulum in its upwards position despite the multiple delays in the plant-control communications.

Due to the noise in the measured angles it is difficult to compare the real with the predicted signals. To see the operation of the predictors, both measured and predicted signals, have been low-pass filtered to remove the noise. These filtered signals are shown in figure 16 for $\theta, \alpha$ and $\gamma$ angles in radians. Although it is not easy to measure because the signals are slightly different, the time between predicted and measured signals is 40,30 and $20 \mathrm{~ms}$, respectively. These are the loop delays between the plant ant the three sub-controllers in the control structure. As the predictors remove the delay influence from the feedbacks, the system behaves as in ideal conditions.

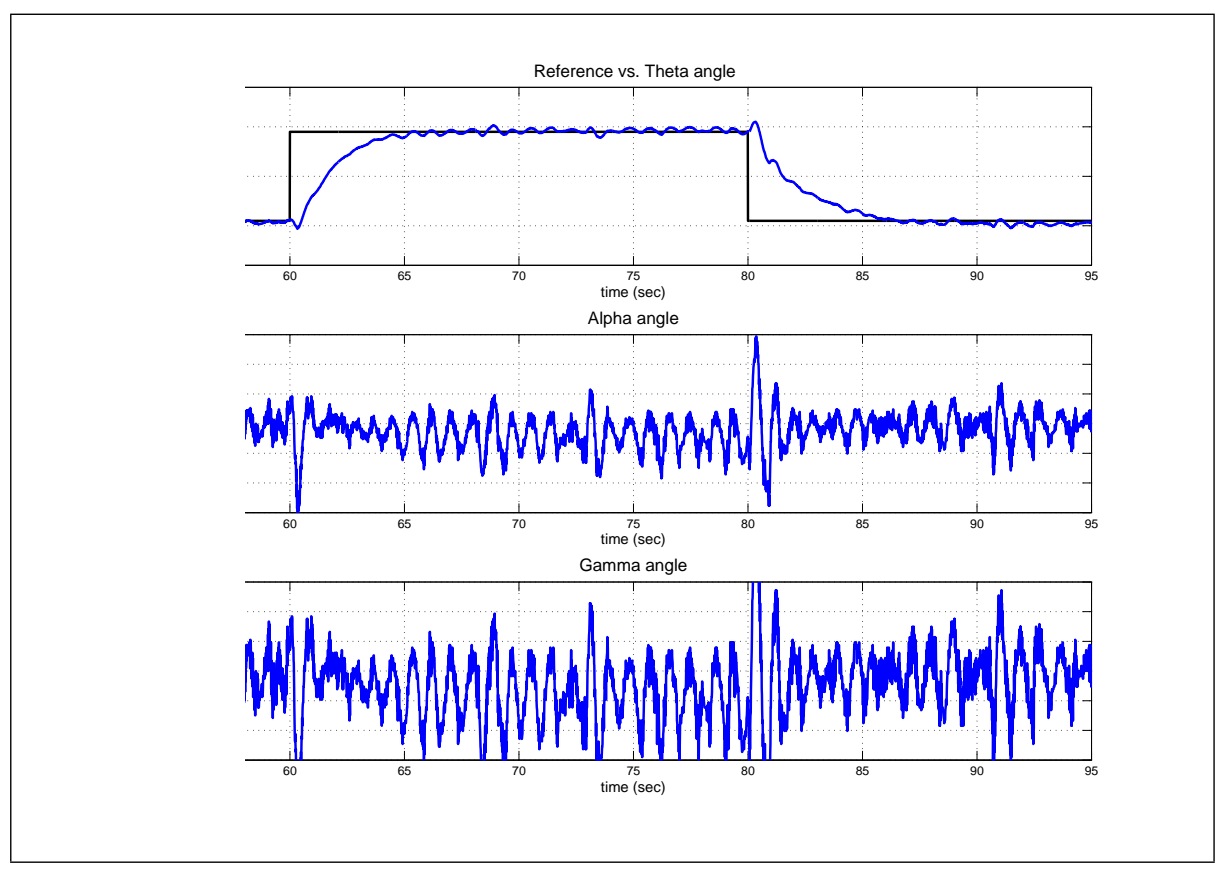

Figure 14: Real results: delayed + compensated.

\section{Conclusions and Future Works}

The aim of this work was to propose a multiloop control structure for a multivariable plant with different delays in each one of the signals between controller and plant. The double inverted rotary pendulum has been chosen for a practical implementation of the proposed control. This 

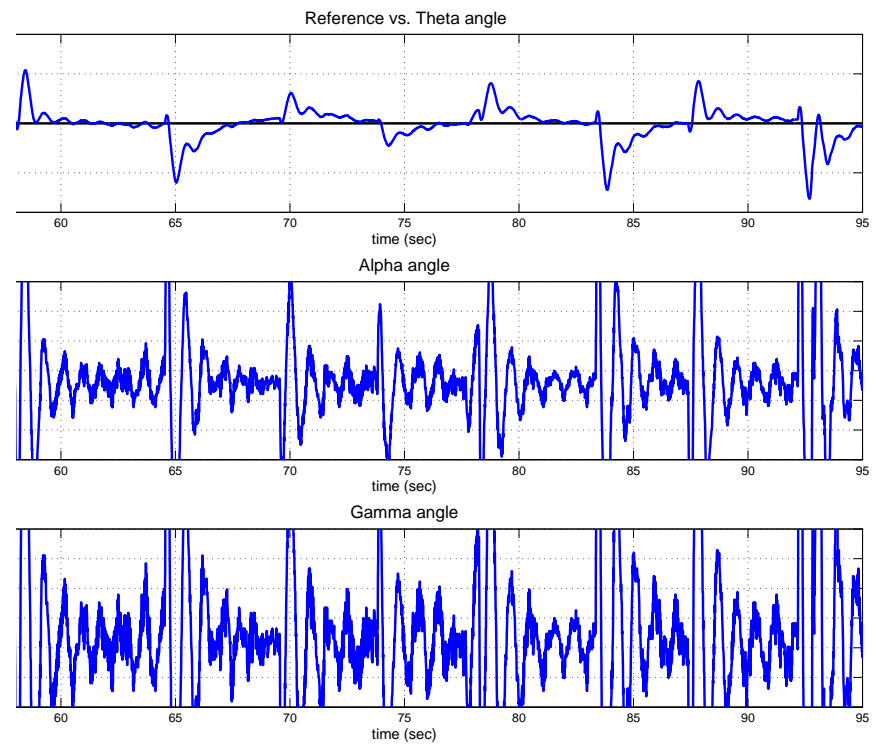

Figure 15: Real results: disturbances.

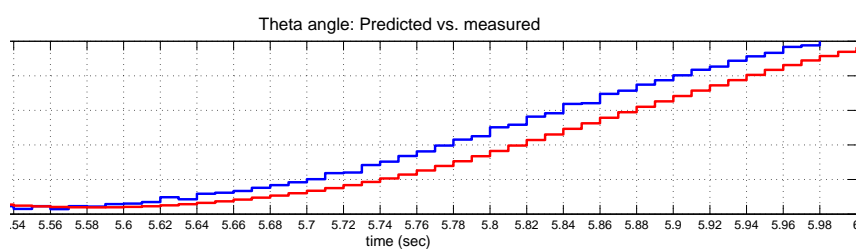

Alpha angle: Predicted vs. measured

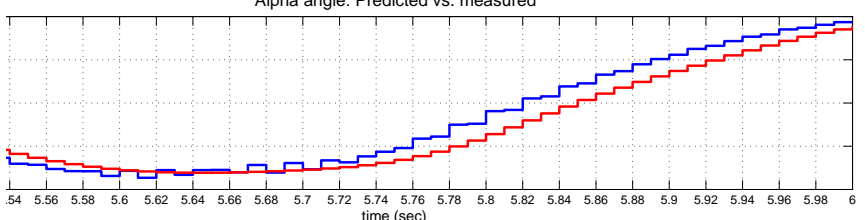

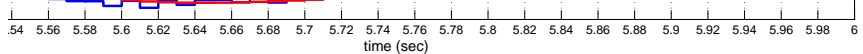

Gamma angle: Predicted vs. measured

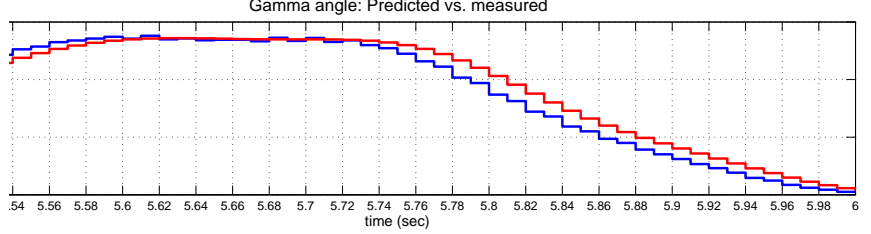

Figure 16: Real results: measured and predicted angles. 
is a classic example of multivariable plant whose stability is seriously worsened even with small delays. There are four links in the system: one control-plant link (actuator) and three plantcontrol ones (sensors). The scenario assumes that this four links are a submitted to different communication delays which increases the difficulty to remove its influence on the stability.

A generalized predictor has been used to provide delay-free information for each one of the three loops in the control structure. Each one has been designed attending to each loop delay. Depending on the quality of the model used in the predictor, the system behaves as in ideal conditions, even with one different delay in each of the links. The validity of the proposed control structure has been shown by means of simulated results. The real implementation using the Quanser double rotary inverted pendulum shows how the proposed structure works when a certain degree of uncertainty, non-negligible noises and disturbances are present.

The delays in this work have been included in the control structure but they should be caused by the communication between sensors/actuators and the device in which the control algorithm is implemented. The goal is to close the loops through a shared communication medium (fieldbus, wireless network...), following different paths for each one of the links. This will cause different transport delays in each loop that may be solved with the proposed structure. In this more realistic implementation an additional problem will arise. The unavoidable lack of synchronization between the devices (sensors, controllers and actuators) will make the delay variable and, probably unknown. Some kind of delay identification must be performed or the control structure must be modified to be adaptive to compensate variable (maybe random) delays.

Another improvement of this proposal is to use a plant with more than one input. This will increase the number of communication links and the number of feedback loops. The 2D inverted pendulum is a good choice to be used in this improvement. This plant increases the degrees of freedom allowing movement in the XY plane. It has two inputs for a planar movement of the cart carrying the pendulum. Two measurements of the cart position must be used in the controller. The rod can bend in two directions giving two angular measurements. So, the plant offers a multivariable model (two inputs, four outputs) and, as is also quite sensitive to delays, is an appropriated plant to extend the work in this paper.

\section{Acknowledgement}

This work was supported by the Spanish Ministerio de Ciencia y Tecnologa Project DPI200914744-C03-03, by Generalitat Valenciana Project GV/2010/018, and by Universidad Polit 'ecnica de Valencia Project PAID06-08.

\section{Bibliography}

[1] Y. Halevi, A. Ray, Integrated communication and control systems: Part I-Analysis, Journal of Dynamic Systems, Measurement and Control, vol. 110, pp. 367-373, 1988.

[2] A. Ray, Y. Halevi, Integrated communication and control systems: Part II-Design considerations, Journal of Dynamic Systems, Measurement and Control, vol. 110, pp. 374-381, 1988.

[3] L.G. Bushnell, Networks and control, IEEE Control Systems Magazine, vol. 21, no. 1, pp. 22-23, 2001.

[4] Y. Tipsuwan, M-Y. Chow, Control methodologies in networked control systems, Control Engineering Practice, vol. 11, no. 10, pp. 1099-1111, 2001. 
[5] P.F. Hokayem, C.T. Abdallah, Inherent issues in networked control systems: A survey, Proceedings of the 23rd American Control Conference, Boston (USA), vol.6, pp. 4897-4902, 2004.

[6] T.C. Yang, Networked control systems: A brief survey, IEE Proceedings on Control Theory and Applications, vol. 153, no. 4, pp. 403-412, 2006.

[7] V. Casanova, J. Salt, Multirate control implementation for an integrated communication and control system, Control Engineering Practice, vol. 11, no. 11, pp. 13351348, 2003.

[8] J. Nilsson, Real-time control systems with delays. PhD thesis, Lund Institute of Technology, Lund, Sweden, 1998.

[9] J. Nilsson, B. Bernhardsson, B. Wittenmark, Stochastic analysis and control of real-time systems with random time delays, Automatica, vol. 34, no. 1, pp. 57-64, 1998.

[10] F.L. Lian, J.R. Moyne, D.M. Tilbury, Performance evaluation of control networks: Ethernet, ControlNet, and DeviceNet, IEEE Control Systems Magazine, vol. 21, pp. 66-83, 2001.

[11] F.L. Lian, J.R. Moyne, D.M. Tilbury, Network design consideration for distributed control systems, IEEE Transactions on Control Systems Technology, vol. 10, pp. 297-307, 2002.

[12] F.L. Lian, J.R. Moyne, D.M. Tilbury, Modelling and optimal controller design of networked control systems with multiple delays, International Journal of Control, vol. 76, no. 6, pp. 591-606, 2003.

[13] H. Gaoa, T. Chenb, J. Lamc, A new delay system approach to network-based control, Automatica, vol. 44, no. 1, pp. 39-52, 2008.

[14] A.V. Savkin, Analysis and synthesis of networked control systems: Topological entropy, observability, robustness and optimal control, Automatica, vol. 42, no. 1, pp. 51-62, 2006.

[15] D.S. Kim et al, Maximum allowable delay bounds of networked control systems, Control Engineering Practice, vol.11, no. 11, pp.1301-1313, 2003.

[16] H. Fanga, H. Yeb, M. Zhong, Fault diagnosis of networked control systems, Annual Reviews in Control, vol.31, no. 1, pp. 55-68, 2007.

[17] O.E. Agamennoni, A.C. Desages, J.A. Romagnoli, A multivariable delay compensator scheme, Chemical Engineering Science, vol. 47, no. 5, pp. 1173-1185, 1992.

[18] B.A. Ogunnaike, W.H. Ray, Multivariable controller design for linear systems having multiple time delays, AIChE Journal, vol. 25, no. 6, pp. 1043-1057, 1979.

[19] K. Watanabe, M. Ito, An observer for linear feedback control laws of multivariable systems with multiple delays in controls and outputs, Systems 83 Control Letters, vol. 1, no. 1, pp. 54-59, 1981.

[20] S. Mori, H. Nishihara, K. Furuta, Control of unstable mechanical system: Control of pendulum, International Journal of Control, vol. 23 no. 5, pp. 673-692, 1976. 
[21] K. Furuta, M. Yamakita, S. Kobayashi, M. Nishimura, A new inverted pendulum apparatus for education, IFAC Advances in Control Education Conference, pp. 191196, 1991.

[22] S. Yurkovich, M. Widjaja, Fuzzy controller synthesis for an inverted pendulum, Control Engineering Practice, vol. 4, no. 4, pp. 455-469, 1996.

[23] W. Zhong, H. Röck, Energy and passivity based control of the double inverted pendulum on cart, IEEE Conference on Control Applications, pp. 896-901, 2001.

[24] J. Driver, D. Thorpe, Design, build and control of a single/double rotational inverted pendulum, The University of Adelaide, School of Mechanical Engineering, Australia, 2004 .

[25] Quanser Consulting Inc, Rotary experiment \#8: Double Inverted Pendulum (DBPEN), 2006.

[26] G. Alevisakis, D.E. Seborg, An extension of the Smith predictor method to multivariable linear systems containing time delays, International Journal of Control, vol. 17, no. 3, pp. 541- 551, 1973.

[27] K. Watanabe, Y. Ishiyama, M. Ito, Modified Smith predictor control for multivariable systems with delays and unmeasurable step disturbances, International Journal of Control, vol. 37, no. 5, pp. 959-973, 1983.

[28] J.M. Maciejowski, Robustness of multivariable Smith predictors, Journal of Process Control, vol. 4, no. 1, pp. 29-32, 1994.

[29] A.M. De Paor, A modified Smith predictor and controller for unstable processes with time delay, International Journal of Control, vol. 41, no. 4, pp. 1025-1036, 1985.

[30] H.J. Kwak, S.W. Sung, I.B. Lee, A modified Smith predictor with a new structure for unstable processes, Industrial \& Engineering Chemistry Research, vol. 38, no. 2, pp. 405-411, 1999.

[31] T. Liu, Y.Z. Cai, D.Y. Gu, W.D. Zhang, New modified Smith predictor scheme for integrating and unstable processes with time delay, IEE Proceedings on Control Theory and Applications, vol. 152, no. 2, pp. 238-246, 2005.

[32] P. García, P. Albertos, T. Hägglund, Control of unstable non-minimum-phase delayed systems, Journal of Process Control, vol. 16, no. 10, pp. 1099-1111, 2006

[33] P. Albertos, P. García, Robust control design for long time-delay systems, Journal of Process Control, vol. 19, no. 10, pp. 1640-1648, 2009. 